\title{
Coincidence Detection of Synaptic Inputs Is Facilitated at the Distal Dendrites after Long-Term Potentiation Induction
}

\author{
Ning-long $\mathrm{Xu},{ }^{1}$ Chang-quan Ye, ${ }^{1} \mathrm{Mu}$-ming Poo, ${ }^{1,2}$ and Xiao-hui Zhang ${ }^{1}$ \\ ${ }^{1}$ Institute of Neuroscience, Shanghai Institutes for Biological Sciences, Chinese Academy of Sciences, Graduate School of Chinese Academy of Sciences, \\ Shanghai 200031, China, and 2Division of Neurobiology, Department of Molecular and Cell Biology, University of California, Berkeley, California \\ 94720-3200
}

Two major aspects of dendritic integration, coincidence detection and temporal integration, depend critically on the spatial and temporal properties of the dendritic summation of synaptic inputs. Neuronal activity capable of inducing synaptic long-term potentiation (LTP) leads to increased linearity of the spatial summation of synchronous EPSPs. Whether such activity can also modulate the temporal summation of EPSPs is unknown. In the present study, we examined the linearity of the summation of EPSPs spaced by different time intervals in hippocampal CA1 pyramidal neurons, before and after LTP induction. We found that LTP induction resulted in an increased linearity of summation of the potentiated input with another synchronous or asynchronous input, with a striking dendritic locationspecific selectivity for the timing of the summed inputs. At distal dendrites, LTP induction led to an increased linearity of summation only for EPSPs arriving within $5 \mathrm{~ms}$, thus favoring the summation of coincident inputs. In contrast, LTP induction at proximal dendrites increased the linearity of summation for EPSPs arriving within a time window of $>20 \mathrm{~ms}$. Furthermore, for synaptic inputs at the distal dendrite, enhanced spiking output after LTP induction was observed only for coincidently summed EPSPs, suggesting facilitated coincidence detection. In contrast, for proximal inputs, enhanced spiking output after LTP induction occurred for EPSPs arriving within a broader time window of $\sim 20 \mathrm{~ms}$, favoring temporal integration. Such dendritic location-dependent differential modulation of coincidence detection and temporal integration by neuronal activity represents a form of activity-dependent and domain-specific plasticity in the function of dendritic information processing.

Key words: dendrite; EPSP summation; coincidence detection; temporal integration; long-term potentiation; LTP; linearity of summation

\section{Introduction}

Most neurons in the mammalian brain receive thousands of excitatory synaptic inputs that are widely distributed along the dendritic arbor and activated with varying degrees of synchronicity. Individual synaptic potentials undergo severe attenuation before reaching the soma (Segev and London, 2000); therefore, spatial and temporal integration of multiple synaptic inputs are critical for the initiation of action potentials (Rall, 1977; Hausser et al., 2000; Magee, 2000; Segev and London, 2000). Dendritic integration is a highly nonlinear process that depends both on passive properties of the dendrite (Rall, 1995; Segev and London, 2000) and on various voltage-gated ion channels (Urban and Barrionuevo, 1998; Cash and Yuste, 1999; Magee, 1999; Hausser et al., 2000; Reyes, 2001; Wang et al., 2003). Recent studies have shown that, in addition to changes in synaptic efficacy, neuronal activity may also induce persistent changes in the property of dendritic

\footnotetext{
Received Dec. 7, 2005; revised Jan. 26, 2006; accepted Jan. 27, 2006.

This work was supported by a grant from the Major State Basic Research Program of China. M.P. was supported in part by a grant from the National Institutes of Health (NS36999). X.Z. was supported by an International Human Frontier Science Program Career Development Award. We thank Dr. Y. Dan for the helpful discussions.

Correspondence should be addressed to either of the following: Xiao-hui Zhang, Institute of Neuroscience, Shanghai Institutes for Biological Sciences, Chinese Academy of Sciences, Shanghai 200031, China, E-mail: xhzhang@ion.ac.cn; or Mu-ming Poo, Department of Molecular and Cell Biology, University of California, Berkeley, CA 94720-3200,E-mail:mpoo@berkeley.edu.

DOI:10.1523/JNEUROSCI.5220-05.2006

Copyright $\odot 2006$ Society for Neuroscience $\quad 0270-6474 / 06 / 263002-08 \$ 15.00 / 0$
}

ion channels (Colbert et al., 1997; Aizenman and Linden, 2000; Bickmeyer et al., 2002; Carr et al., 2003; Wang et al., 2003; Zhang and Linden, 2003; Frick et al., 2004) and the morphology of dendritic spines (Engert and Bonhoeffer, 1999; Maletic-Savatic et al., 1999; Toni et al., 1999; Yuste and Bonhoeffer, 2001; Zhou et al., 2004). Thus, dendritic integration of synaptic inputs is likely to be regulated by neuronal activity as well. A recent study (Wang et al., 2003 ) indeed revealed bidirectional changes in the linearity of the spatial summation of EPSPs accompanying long-term potentiation (LTP) and long-term depression (LTD) induced by correlated presynaptic and postsynaptic activity.

Two fundamental aspects of dendritic integration of synaptic inputs are coincidence detection and temporal integration (Segev, 1995; Magee, 2000), both of which are essential for neuronal information processing (Shadlen and Newsome, 1994; Segev, 1995; Konig et al., 1996; Agmon-Snir et al., 1998). Coincident detection is reflected by the firing of the postsynaptic neuron when spatially separated inputs are activated synchronously at the dendrite, whereas temporal integration depends on the efficacy of EPSP summation resulting from sequential activation of the same input or by asynchronous activation of different inputs. Thus, a selective enhancement of summation of synchronous and asynchronous inputs will promote coincidence detection and temporal integration, respectively. Furthermore, the cable properties of dendrites (Rall, 1977) predict that temporal integration 
A

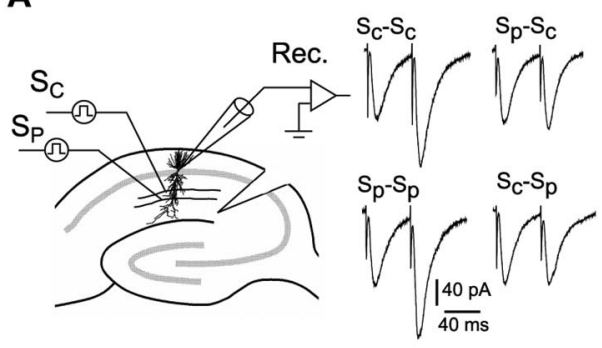

C

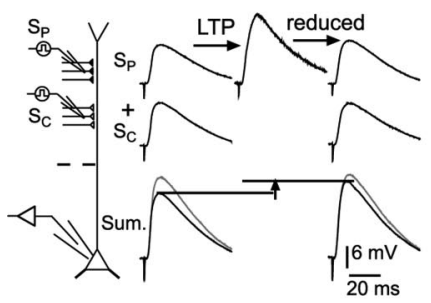

D

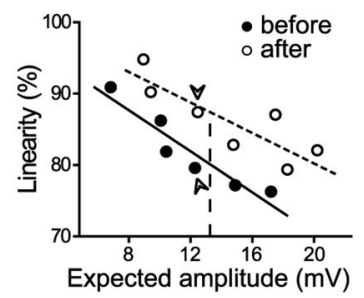

B
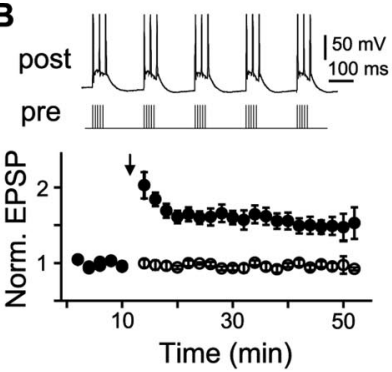

E

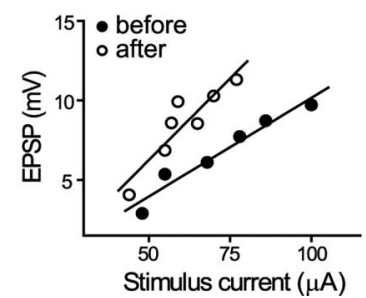

Figure 1. Increased linearity of summation for two distal SC inputs after LTP induction. $\boldsymbol{A}$, Schematic diagram depicting two independent Schaffer collateral inputs stimulated by extracellular electrodes. One of the inputs was conditioned by pTBS (see Materials and Methods). Sample traces depict EPSCs elicited by sequential stimulation of the same input (Sc-Sc and Sp-Sp) or two inputs $\left(S_{p}-S_{c}\right.$ and $\left.S_{c}-S_{p}\right)$ at an interval of $50 \mathrm{~ms}$, showing the presence of pair-pulsed facilitation at the same input and absence of cross-facilitation between two stimulated pathways. $\boldsymbol{B}$, Persistent increase in EPSP amplitude ( $164.5 \pm 9.3 \%$ of the mean value before induction; $n=9$ ) was induced at the input exposed to pTBS ( $S_{p} ;$ illed circles) but not at the control input ( $S_{c}$; open circles). Traces above depict pTBS protocol. $C$, Increased linearity for two synchronous inputs at a distal dendrite. Left, Schematic diagram depicting the location of $L T P$ induction $\left(S_{P}\right)$ and an adjacent control input $\left(S_{C}\right)$. The dashed line marks $150 \mu \mathrm{m}$ from the soma. Right, Top traces, Sample EPSPs from $S_{P}$, showing the increase in amplitude after LTP induction and subsequent adjustment back to the control level. Middle traces, Sample EPSPs from $S_{C}$, showing no change in amplitude. Bottom traces, Samples of summed EPSPs elicited by synchronous and asynchronous stimulation of $S_{p}$ and $S_{c}$ before and after LTP induction. The linearity of summation was defined as the ratio (in percentage) of measured amplitude of summed EPSPs (dark traces) to the expected amplitude (arithmetical sum; gray traces) calculated from two individual EPSPS. D, Linearity versus expected amplitude of summed EPSPs from one experiment before (filled circles) and after (open circles) LTP induction. Arrowheads mark the data shown in C.E, Typical case in which EPSP amplitude was varied by adjusting the stimulus intensity. Synaptic potentiation was indicated by leftward shift of the stimulus intensity-EPSP amplitude curve. Lines represent the best linear fit. Rec., Recording pipette; Norm., normalized; post, postsynaptic; pre, presynaptic.

of EPSPs is more pronounced for distal inputs because of their prolonged time course and attenuated amplitude of EPSPs when reaching the soma, whereas coincidence detection is more prominent for proximal inputs (Rall, 1977; Segev, 1995; Magee, 2000). Thus, it is important to understand the temporal characteristics of the modification of dendritic summation of EPSPs by neuronal activity and its dependence on the dendritic location of the inputs. In the present study, we examined the modification of dendritic summation of synaptic inputs arriving with various degrees of synchronicity and at different dendritic locations after LTP induction. In addition, by monitoring the spiking output of the summed EPSPs, we investigated the consequence of this activity-dependent modification of EPSP summation on the coincidence detection and temporal integration of the neuron for inputs arriving at different dendritic locations.

\section{Materials and Methods}

Slice preparation. Hippocampal slices $(350-400 \mu \mathrm{m}$ thick) were prepared from Sprague Dawley rats (postnatal days 16-20), as described previously (Wang et al., 2003). Neurons were visualized with an Olympus microscope (BX51WI) equipped with an infrared video camera and differential interference contrast optics. The extracellular solution contained the following (in mM): $119 \mathrm{NaCl}, 2.5 \mathrm{KCl}, 2.5 \mathrm{CaCl}_{2}, 1.3 \mathrm{MgSO}_{4}, 1$ $\mathrm{NaH}_{2} \mathrm{PO}_{4}, 26.2 \mathrm{NaHCO}_{3}$, and 11 glucose, saturated with $95 \% \mathrm{O}_{2}$ and $5 \%$ $\mathrm{CO}_{2}, \mathrm{pH} 7.4 ; 28-30^{\circ} \mathrm{C}$. All experiments were performed in the presence of picrotoxin $(100 \mu \mathrm{M})$. The inputs from area CA3 were eliminated by a cut of the slice between CA3 and CA1 to prevent repetitive firing caused by reduced GABAergic inhibition.

Electrophysiology. Whole-cell recordings were made with amphotericin $\mathrm{B}(0.3 \mathrm{mg} / \mathrm{ml}$; Calbiochem, La Jolla, CA) perforation on the soma of CA1 pyramidal neurons. Recording pipettes were filled with an internal solution containing the following (in $\mathrm{mm}$ ): 136.5 K-gluconate, $17.5 \mathrm{KCl}, 9.0 \mathrm{NaCl}, 1.0 \mathrm{MgCl}_{2}$, 10.0 HEPES, and 0.2 EGTA, pH 7.3, with pipette resistance of 2-4 M $\Omega$. Series resistance ranged from 15 to $40 \mathrm{M} \Omega$ and became stable 5-15 min after seal formation. The absence of break-in during the perforated-patch recording was confirmed by the stability of the membrane potential when tetraethylammonium (5 $\mathrm{mm})$ was added to the intracellular solution $(n=8)$. The cell was usually current clamped at $-70 \mathrm{mV}$ by a constant current injection throughout the experiment, and monosynaptic EPSPs were evoked by focal extracellular stimulation $(100 \mu \mathrm{s} ; 10-50 \mu \mathrm{A})$ with small glass electrodes (tip opening, $2-3 \mu \mathrm{m}$; placed within $30 \mu \mathrm{m}$ of the main dendrite) by means of a stimulator (Master-8; A.M.P.I., Jerusalem, Israel) coupled through an isolator (Iso-flex; A.M.P.I.). Data were amplified and filtered at 2 $\mathrm{kHz}$ by a patch-clamp amplifier (Axopatch 200B), digitalized (DIGIDATA 1322A), stored, and analyzed by pCLAMP (Molecular Devices, Union City, CA). Data were discarded when the input resistance changed $>20 \%$ during recording, which is often accompanied by an increase of series resistance, indicating a deterioration of patch configuration. The dendritic locations selected for the present study $(60-300 \mu \mathrm{m})$ were chosen for the purpose of obtaining EPSPs of a substantial range of amplitudes. For stimulating two Schaffer collateral-commissural (SC) inputs, the interpipette distance was in a range of 30 to $80 \mu \mathrm{m}$. A paired-pulse test (with a $50 \mathrm{~ms}$ interval) was applied under the voltage-clamp mode to confirm the independence of the two inputs by the absence of crossfacilitation $(n=9)$ (see Fig. $1 A$ ). For examining the linearity of EPSP summation, the summed and individual EPSPs at each stimulus strength were recorded for 10 trials at $0.04 \mathrm{~Hz}$, and averaged traces were used for calculating the linearity. The linearity of summation was defined as the ratio of the peak amplitude of summed EPSPs to that of algebraically summed traces (Cash and Yuste, 1999; Wang et al., 2003). Data are presented as mean \pm SEM, and statistical significance was tested with the paired Student's $t$ test, unless stated otherwise. For the induction of LTP, paired theta-burst stimulation (pTBS) consisted of five paired spike trains in both presynaptic and postsynaptic neurons at the theta frequency $(5 \mathrm{~Hz})$. Each pair of spike trains consisted of five presynaptic spikes at $100 \mathrm{~Hz}$ and three postsynaptic spikes at $40 \mathrm{~Hz}$, with synchronous onset of the first spike. Application of two pTBSs spaced $10 \mathrm{~s}$ to the hippocampal SC-CA1 pathway was effective in inducing LTP (see Fig. 1B) (Magee and Johnston, 1997). The EPSP amplitude before the induction was typically $4-6 \mathrm{mV}$. The summation test was resumed at least 15 min after LTP induction and lasted for at least $40 \mathrm{~min}$. All other chemicals were from Sigma (St. Louis, MO).

\section{Results}

Measurements of dendritic summation of EPSPs

Perforated whole-cell recordings were made from the soma of CA1 pyramidal neurons in acute hippocampal slices to monitor monosynaptic EPSPs, which were elicited by extracellular stimulation of two separate SC fibers in the stratum radiatum (Fig. $1 A$ ) 
(see Materials and Methods), in the presence of the $\mathrm{GABA}_{\mathrm{A}}$ receptor antagonist picrotoxin $(100 \mu \mathrm{M})$. To induce robust LTP, we used pTBS (see Materials and Methods), which induces a persistent increase in EPSP amplitude only at the conditioned input $\left(\mathrm{S}_{\mathrm{P}}\right)$ but not at the control input $\left(\mathrm{S}_{\mathrm{C}}\right)$ (Fig. $1 B$ ). Summation of EPSPs was examined by costimulation of two independent (nonoverlapping) SC inputs (with stimulating electrodes separated by $\sim 50 \mu \mathrm{m}$ ) at various interstimulus intervals $(\Delta t)$. The independence of $S_{P}$ and $S_{C}$ inputs was confirmed by the absence of crossfacilitation between EPSPs elicited by sequential stimulation to $S_{P}$ and $S_{C}$ with an interval of $50 \mathrm{~ms}$; however, when the same test was applied to either $S_{P}$ or $S_{C}$, apparent pair-pulse facilitation was usually observed at these SC-CA1 synapses (Fig. $1 A)$. The linearity is defined as the ratio between the peak amplitude of the recorded response induced by costimulation and that of the algebraic summation ("expected sum") calculated from the amplitude of two individual EPSPs. An example is shown in Figure $1 C$ for two synchronous inputs at the distal dendrite. To compare summation linearity over the same range of amplitudes of the expected sum, we adjusted the EPSP amplitude of the potentiated input back to the control level by reducing the stimulus strength at $\mathrm{S}_{\mathrm{P}}$ after LTP induction (Fig. 1C,D). Consistent with previous studies (Urban and Barrionuevo, 1998; Cash and Yuste, 1999; Wang et al., 2003), the linearity of summation depended on the EPSP amplitude (i.e., larger EPSPs sum with a lower linearity) (Fig. 1D). The success of LTP induction was also reflected by the reduced stimulus strength required to elicit the same amplitude of EPSPs (Fig. 1E).

\section{A narrow time window for increased linearity of summation of distal inputs}

The apical dendrite of pyramidal neurons exhibits marked heterogeneity along the somatodendritic axis in both the passive and active properties, leading to the dendrite-location dependence of dendritic signal processing (Hoffman et al., 1997; Magee, 1998; Cash and Yuste, 1999; Segev and London, 2000; Williams and Stuart, 2002) and synaptic plasticity (Froemke et al., 2005). We first examined changes in the summation of two EPSPs arriving at different intervals at the distal dendrite (160-300 $\mu \mathrm{m}$ from the soma) before and after LTP induction at one of the two inputs (with $S_{P}$ more distal than $S_{C}$ ). After the application of pTBS, which successfully induced LTP, we found a significant increase in the linearity of summation for synchronous EPSPs. To summarize the results, an increase in linearity at an amplitude of 13 $\mathrm{mV}$ was estimated (Fig. $1 D$, dashed line) for all cells before and after LTP induction (Fig. $2 A)(0 \mathrm{~ms} ; 6.5 \pm 0.9 \%$ increase; $p=$ $0.0005 ; n=7$ ), consistent with a previous report (Wang et al., 2003); however, when the two inputs were stimulated at different intervals, we found a slight but significant increase only at the interval of $5 \mathrm{~ms}(4.3 \pm 0.8 \%$ increase; $p=0.001 ; n=8)$ and not at larger intervals of 10 or $15 \mathrm{~ms}(p>0.5)$ (Fig. $2 A)$. Similar results were found when the location of $S_{P}$ and $S_{C}$ was switched (Fig. $2 B$ ). Thus, the distal dendrites exhibited a narrow temporal window for the increase in linearity of summation, with enhancement only for summation of the potentiated input with other coincidently arriving EPSPs after LTP induction.

\section{A broader time window for increased linearity of summation of proximal inputs}

We further examined the linearity of summation of two proximal SC inputs (60-140 $\mu \mathrm{m}$ from the soma) arriving at different intervals before and after LTP induction in one of the two inputs. When $S_{P}$ was more proximal to $S_{C}$, we found a significant increase in the summation linearity for EPSP intervals up to $20 \mathrm{~ms}$ after LTP induction at $S_{\mathrm{P}}$, regardless of the temporal order of the two inputs (Fig. $3 A$ ). Similar results were found when the location of $S_{P}$ and $S_{C}$ was switched (Fig. $3 B$ ). This broader time window for summation enhancement is comparable with the characteristic decay time of EPSPs $(37.5 \pm 3.4 \mathrm{~ms})$.

The results on the temporal specificity for the enhancement of summation linearity are summarized in Figure $4 A$, which shows distinct time windows for distal versus proximal inputs. As shown in Figure $4 B$, the distribution of the dendritic location of $S_{P}$ in all experiments covers two distinct ranges of distance from the soma. It is unlikely that the location-dependent temporal windows were caused by differences in LTP induction, because the average magnitudes of LTP induced at distal versus proximal inputs, as reflected in the percentage increment of EPSP amplitude at 15-30 min after pTBS application, was not significantly different between the two groups (distal: $78.8 \pm 9.2 \%, n=20$; proximal: $88.1 \pm 6.7 \%, n=22 ; p=0.43$; Student's $t$ test). 


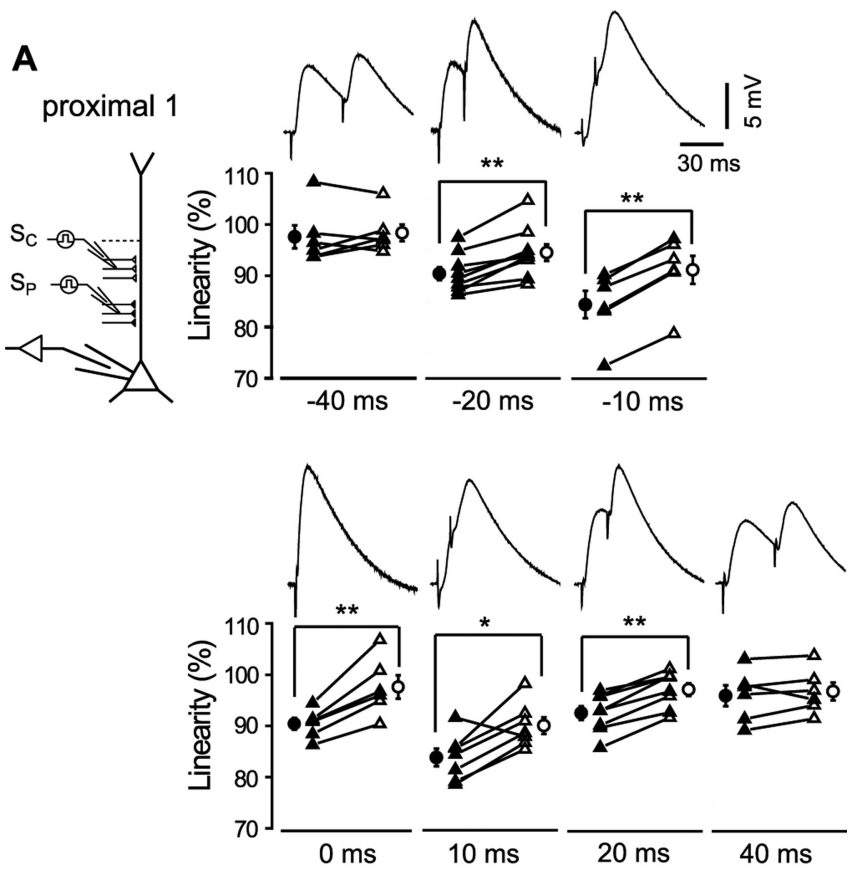

B

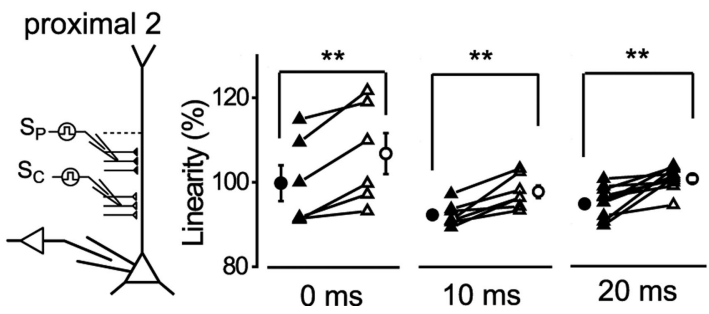

Figure 3. Temporal selectivity of pTBS-induced increase in the linearity of summation of two proximal SC inputs. A, Summation linearity for two proximal inputs before and after LTP induction at one of the inputs, with $S_{p}$ proximal to $S_{C}$ (proximal 1 ; schematic diagram on left). Top traces depict samples of summed EPSPs for two consecutive EPSPs at different intervals. Data below show the linearity before and after LTP induction (as in Fig. $2 A$ ). Error bars indicate SEM. Negative intervals denote $S_{C}$ preceding $S_{p} . B$, Similar to $A$ except that $S_{p}$ is distal to $S_{C}$ (proximal 2). Data with significant increase are marked $\left({ }^{*} p<0.05 ;{ }^{* *} p<0.01\right)$.

\section{Enhanced coincidence detection for distal inputs after LTP}

The temporal characteristics of dendritic summation of EPSPs are likely to influence the coincidence detection and temporal integration of a neuron. Thus, the different temporal specificity in the increase of summation linearity described above may differentially promote these two modes of integration at the distal versus proximal dendrites. To test this idea, we examined the probability of spiking triggered by two summed EPSPs at different intervals before and after LTP induction. Stimuli of relatively high intensity were delivered to elicit summed EPSPs with peak amplitudes close to the firing threshold. To eliminate the effect of the increased EPSP amplitude after LTP induction, the stimulus strength of $S_{\mathrm{P}}$ was reduced to elicit EPSPs of amplitudes similar to those observed before LTP induction (Fig. 5A).

In the first set of experiments, we examined the integration of two distal SC inputs. During the control period, the stimulus strength was set so that individual EPSPs elicited by $S_{C}$ and $S_{P}$ were subthreshold and similar in amplitude, and the synchronous summation $(\Delta t=0 \mathrm{~ms})$ triggered spikes in half of the 15 trials (Fig. 5B, left). As shown by the example in Figure 5B, spiking was also triggered frequently by two asynchronous EPSPs over a broad range of intervals in the presence of picrotoxin, consistent with a poor coincidence detection described in previous reports under the condition of reduced inhibition (Pouille and Scanziani, 2001; Lamsa et al., 2005); however, after LTP induction and subsequent reduction of the stimulus strength at $\mathrm{S}_{\mathrm{P}}$ (Fig. $5 A, B$ ), an increase in the spiking probability was observed near $\Delta t=0 \mathrm{~ms}$, whereas that for longer intervals remained largely unchanged. Results from four experiments (with $S_{P}$ distal to $S_{C}$ ) are summarized in Figure 5C. Enhanced spiking was found only for near-synchronous summation but not for summation with $\Delta t \geq 6 \mathrm{~ms}$, suggesting that coincidence detection was selectively enhanced after LTP induction. The same effect was observed when $S_{P}$ was proximal to $S_{C}$ (data not shown). That the spiking probability for asynchronous EPSPs was not enhanced after LTP induction was further confirmed by the finding that there was no significant change in the spiking output elicited by two asynchronous EPSPs ( $\Delta t=10 \mathrm{~ms}$ ) of a wide range of EPSP amplitude after LTP induction (Fig. 5D). The narrow window for the enhancement of spiking output of the neuron triggered by two distal inputs corresponds to that found for the increase in summation linearity observed for these inputs after LTP induction, suggesting that the latter may cause the enhanced coincidence detection between the potentiated input and other distal input.

Enhanced temporal integration for proximal inputs after LTP Spiking probability tests were also performed for two proximal SC inputs to the CA1 pyramidal cell. In contrast to that found for distal inputs, spikes were triggered only by EPSPs arriving within a much narrower time window before LTP induction (Fig. 6A, left) as a result of faster kinetics of EPSPs elicited at proximal inputs (data not shown) (Magee and Cook, 2000). After LTP induction at one of the two proximal inputs, however, the spiking probability increased for both synchronous and asynchronous EPSPs with intervals up to $18 \mathrm{~ms}$ (Fig. $6 A, B)(n=6)$, indicating an overall enhancement of temporal integration of EPSPs. This enhancement of spiking probability was further confirmed by examining the spiking output of asynchronous EPSPs $(\Delta t=10$ $\mathrm{ms}$ ) for a wide range of EPSP amplitude (Fig. $6 \mathrm{C}$ ). The broad temporal window for the enhancement of temporal integration is similar to that found for the increase in summation linearity for proximal inputs. Thus the distal-proximal difference in the temporal specificity for increases in linearity after LTP induction may differentially enhance coincidence detection and temporal integration for distal and proximal inputs, respectively.

\section{Enhanced temporal summation at single inputs after LTP induction}

Because of more elaborate arborization and heterogeneity in channel distribution along the apical dendrite (Bannister and Larkman, 1995; Hoffman et al., 1997; Magee, 1998; Migliore and Shepherd, 2002), distal dendrites are electrically more compartmentalized than proximal dendrites (Segev and London, 2000), and the local EPSP at the distal dendrites decays more rapidly (Magee, 2000). Thus, local interaction between spatially segregated distal synapses is likely to be restricted within a narrower time window. This effect, together with localized modification of dendritic properties (Wang et al., 2003; Frick et al., 2004), may lead to a narrower time window for enhancement of summation at distal dendrites. To test this possibility, we eliminated the effect of compartmentalization on spatially segregated distal inputs by delivering sequential stimuli $(\Delta t=10 \mathrm{~ms})$ to the same input, eliciting two EPSPs with an interval of $10 \mathrm{~ms}$ (Fig. 7A, left). In contrast to that found for two separate inputs (Fig. 2), we observed a significant increase in the linearity of summation for 
sequential EPSPs at the single distal input $(7.3 \pm 1.2 \% ; p=0.0002 ; n=10)($ Fig. $7 A)$ after LTP induction at the same input, suggesting that a linearity increase does occur for longer time intervals when there is less spatial segregation of synapses. Such enhancement of temporal summation was unlikely to result from an enhanced paired-pulse facilitation (caused by a reduced presynaptic release probability), because LTP induction in these CA1 pyramidal neurons rarely leads to such enhancement (Bolshakov and Siegelbaum, 1995; Nicoll and Malenka, 1999). Furthermore, the spiking probability for two sequential EPSPs at the single input was significantly elevated after LTP induction for both distal (Fig. $7 B$ ) and proximal (Fig. 7C) inputs, in sharp contrast to that found for two separate asynchronous inputs activated at the same $10 \mathrm{~ms}$ interval (Fig. 2D). This is consistent with the idea that activity-induced differential enhancement in dendritic integration results from a differential effect of spatial segregation of inputs along the apical dendrite. Finally, in support of the idea of localized dendritic modification, we found that after LTP induction at a distal input, there was no increase in linearity for the summation of two sequential EPSPs (interval 10 $\mathrm{ms}$ ) at an adjacent control input 50-80 $\mu \mathrm{m}$ away, either more proximal (Fig. 7D) or more distal than the potentiated input (Fig. $7 E)$. These results on the temporal summation at single inputs are consistent with the notion that the distal-proximal difference in the temporal specificity in the enhancement of dendritic integration may be determined by the extent of local synaptic interaction at distal versus proximal dendrites.

\section{Discussion}

In this study, we have examined the modification of dendritic summation of EPSPs, which are elicited with different degrees of synchronicity at different dendritic locations after the induction of LTP by correlated presynaptic and postsynaptic bursting activities. The functional consequence of such a modification was analyzed for two aspects of neuronal operation: coincidence detection and temporal integration. By varying the dendritic location and the interval between the summed EPSPs, we discovered a striking dendritic location-dependent temporal specificity in the enhancement of dendritic summation after LTP induction. For distal synaptic inputs, LTP induction led to an increased linearity of summation only for the summation of potentiated input with other coincident SC inputs, whereas for proximal inputs, the increase in linearity occurred for the summation of inputs arriving within a much broader temporal window. We showed further, by monitoring the spike generation in the neuron, that such a distal-proximal difference for the increase in summation linearity could account for differential enhancement of coincidence detection and temporal integration for distal and proximal inputs, respectively.

Input specificity in the enhancement of dendritic summation A previous study on hippocampal slices (Wang et al., 2003) has shown that LTP induction at SC-CA1 pyramidal cell synapses leads to a persistent enhancement of dendritic summation of two synchronous nonoverlapping SC inputs, but only for summation involving the potentiated input. This input specificity in the
$\Delta t(\mathrm{~ms})$

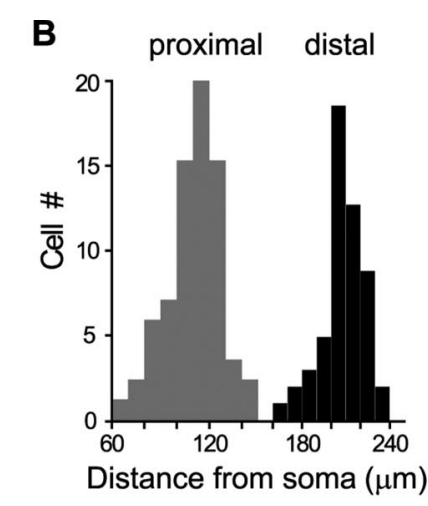

tion of distal versus proximal SC inputs after LTP induction. $\boldsymbol{A}$, Summarized data on the changes in linearity versus the interval between the two EPSPS elicited at $S_{P}$ and $S_{C}$ of and $S_{C}$ are located at either the distal $(160-300 \mu \mathrm{m})$ or proximal $(60-140 \mu \mathrm{m})$ dendrite. $\boldsymbol{B}$, Distribution of the location of $S_{P}$ relative to the soma in all experiments shown in $\boldsymbol{A}$. prox., Proximal.

activity-induced modification of dendritic integration was further confirmed in the present study by our findings that there is no change in the linearity of temporal summation of sequential EPSPs at single inputs adjacent to the potentiated input (Fig. $7 D, E)$. It is well known that LTP induction in the hippocampus is largely input specific (i.e., only synapses experiencing the LTPinducing stimulation become potentiated) (Brown et al., 1990; Malenka and Nicoll, 1999; Bi and Poo, 2001). This input specificity in synaptic modification is an essential element of the Hebb's learning rule, which has been implemented successfully in models of unsupervised learning networks and experiencedependent refinement of neural circuits. In addition to LTP, input specificity has also been demonstrated in the activity-induced potentiation of EPSP-spiking coupling, a phenomenon that depends on changes in local dendritic ion conductances (Daoudal et al., 2002). The activity-induced enhancement of dendritic integration, which occurred only for summation involving the potentiated input, will elevate the power of the summed inputs in controlling the spiking output of the postsynaptic neuron without compromising the input specificity, thus preserving the information fidelity of the potentiated input.

\section{Potential mechanisms for the distal-proximal difference in temporal specificity}

The present study revealed a striking distal-proximal difference in the temporal specificity of the enhancement of dendritic integration. The underlying mechanism for this distal-proximal difference is yet to be elucidated. One possibility is that LTP induction may generate differential effects on local dendritic properties at various dendritic locations. Local modulation of $I_{\mathrm{h}}$ channels and NMDA receptors has been suggested to be responsible for the changes in summation linearity after LTP induction (Wang et al., 2003), and local modulation of $I_{\mathrm{A}}$ channel kinetics after LTP induction has been shown to facilitate action potential back propagation (Frick et al., 2004). Both $I_{\mathrm{h}}$ and $I_{\mathrm{A}}$ channels are expressed with increasing gradient from the soma to the distal optical dendrites (Hoffman et al., 1997; Magee, 1998; Migliore and Shepherd, 2002). The expression of NMDA receptors and the distribution of glutamatergic synapses are also highly heterogeneous along the apical dendrites. Such heterogeneity in channel and receptor distribution thus may result in a quantitative difference in the changes of dendritic properties after LTP induction, 

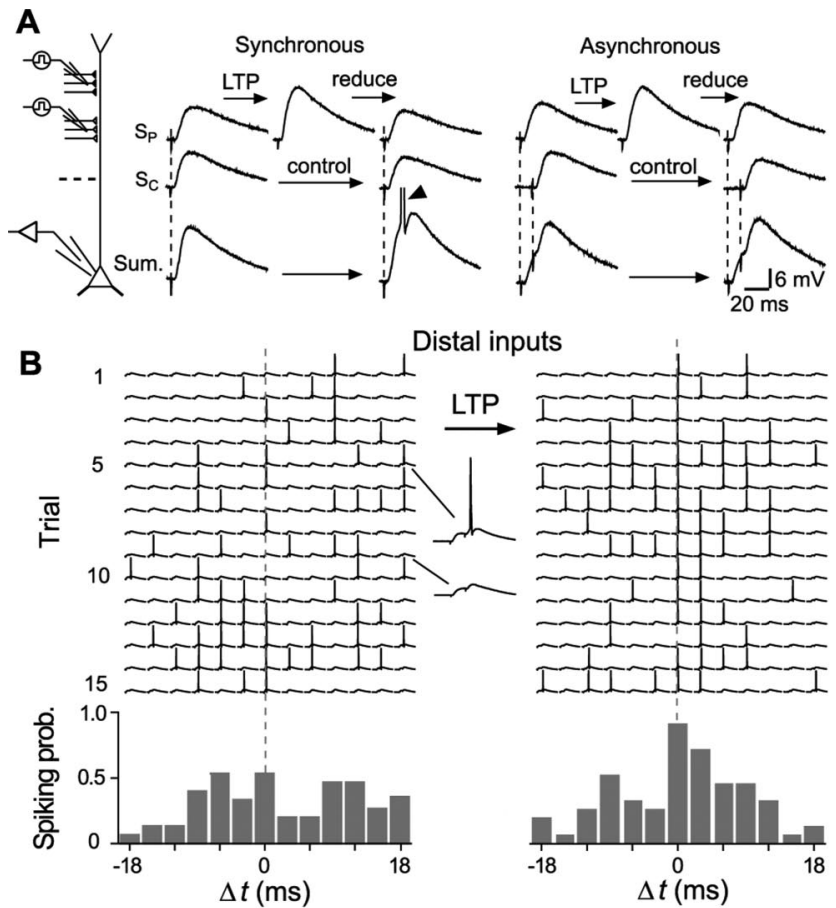

Distal inputs
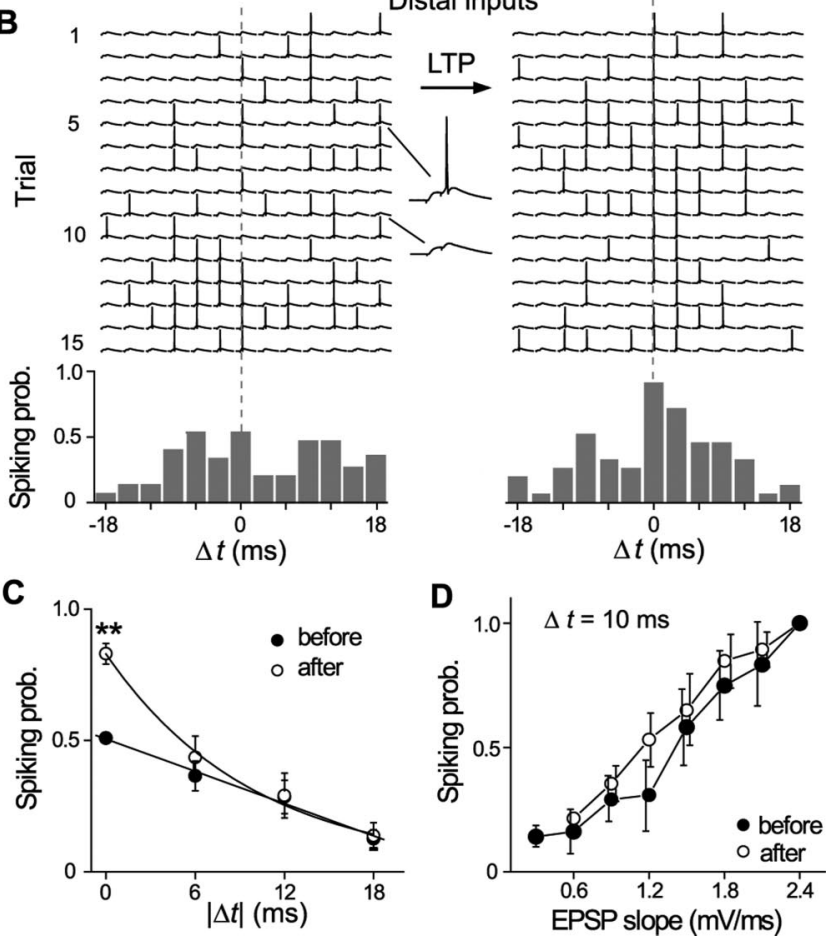

Figure 5. LTP induction facilitated coincidence detection for distal inputs. $A$, Protocols for assessing spiking probability elicited by two synchronous or asynchronous (interval, $10 \mathrm{~ms}$ ) EPSPs before and after LTP induction. Schematic diagram on the left depicts two independent distal SC inputs (200-300 $\mu \mathrm{m}$ from soma). Top traces, Sample EPSPs from $S_{p}$, showing the increase in amplitude after LTP induction and subsequent adjustment back to the control level. Middle traces, Sample EPSPs from $S_{C}$, showing no change in amplitude. Bottom traces, Samples of summed EPSPs elicited by synchronous and asynchronous stimulation of $S_{p}$ and $S_{C}$ before and after LTP induction, with spiking (arrowhead, truncated) induced by synchronous summation after LTP. B, Example experiment showing spike initiation by summed EPSPs elicited at two distal inputs at different interstimulus intervals before (left) and after (right) LTP induction. Traces above depict summed responses for two $\mathrm{SC}$ inputs stimulated at 13 different intervals $(\Delta t=-18$ to $+18 \mathrm{~ms})$, recorded from 15 consecutive trials before and after LTP induction. The traces with $\Delta t=18$ at trials 5 and 9 of the control period are magnified. Dashed lines indicate synchronous summation ( $\Delta t=0 \mathrm{~ms}$ ). Histograms below show probability of spiking at different intervals for the 15 trails shown above. Note that the probability at $\Delta t=0 \mathrm{~ms}$ was set at $\sim 0.5$ before LTP induction by adjusting the initial EPSP amplitudes. After LTP induction, the stimulus strength was reduced so that the EPSP amplitude of $S_{p}$ was the same as the previous LTP induction. $C$, The average spiking probability ( \pm SEM) for summed EPSPs at $\Delta t=$ $0, \pm 6, \pm 12$, and $\pm 18 \mathrm{~ms}$ before and after LTP induction. Solid lines represent the best firstorder exponential fit. (** $p=0.0085 ; n=4$ ). $\boldsymbol{D}$, Probability of spiking elicited by stimulating two SC inputs at distal dendrites plotted against the EPSP slope of $S_{p}$ (summation interval, 10 $\mathrm{ms})$. The strength of $S_{p}$ was adjusted to elicit EPSPs of different amplitude, whereas that of $S_{C}$ was kept constant. The data were sorted into bins of EPSP slope of $S_{P}$ (bin size, $\left.0.3 \mathrm{mV} / \mathrm{ms}\right)$. No significant difference was found between the data sets before and after LTP induction ( $p=$ 0.15; Mann-Whitney $U$ test). prob., Probability.

leading to distal-proximal differences in the enhancement of dendritic integration.

This distal-proximal difference may also be attributed to differences in the local synaptic interaction resulting from differen-
A

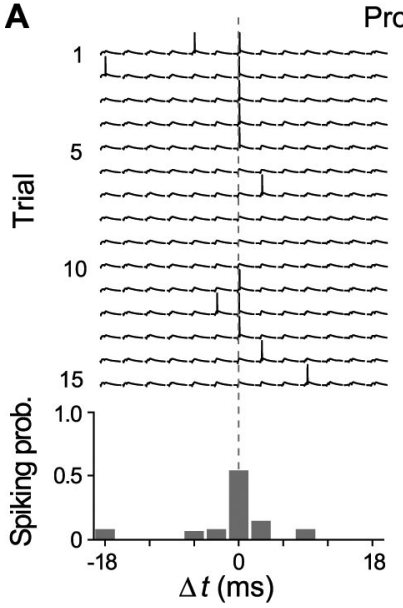

Proximal inputs

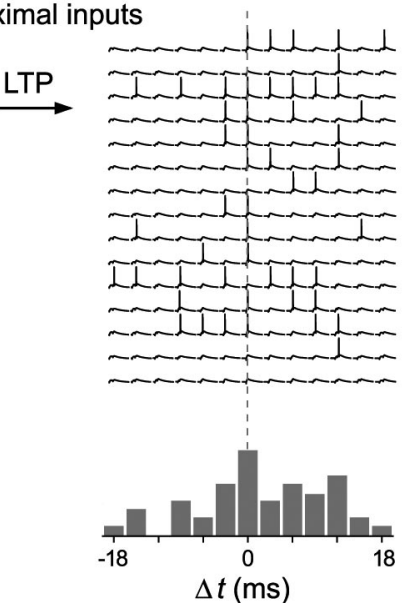

B

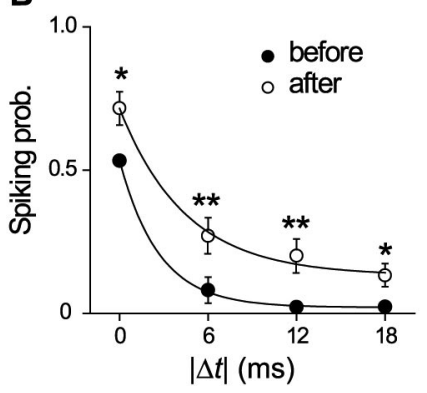

C

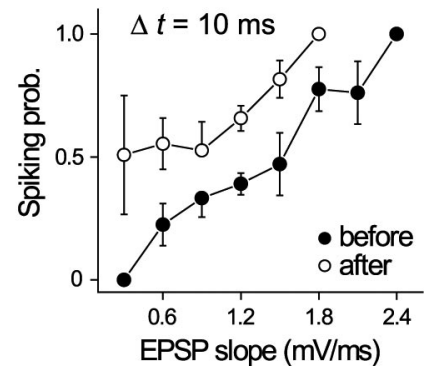

Figure 6. LTP induction enhanced temporal integration for proximal inputs. $\boldsymbol{A}$, Example experiment showing spike initiation by summed EPSPs elicited at two proximal inputs at different interstimulus intervals before (left) and after (right) LTP induction. Data are presented in the same manner as in Figure $5 A$. $\boldsymbol{B}$, Summary from all experiments on spike initiation elicited by stimulating two proximal inputs before and after LTP induction ${ }^{*} p<0.05 ;{ }^{* *} p<0.01 ; n=$ 6). C, Probability of spiking elicited by two proximal inputs plotted against the EPSP slope of $S_{P}$ (summation interval, $10 \mathrm{~ms}$ ). A significant difference was found between the data sets before and after LTP induction ( $p=0.001$; Mann-Whitney $U$ test). prob., Probability.

tial arborization of distal versus proximal dendrites. The complexity in the arborization of an apical dendrite of a pyramidal neuron increases with the distance from the soma (Bannister and Larkman, 1995), resulting in more extensive electrical compartmentalization of synapses (Segev and London, 2000) and more rapid decay of local EPSPs (Magee, 2000) at the distal dendrite. Local synaptic interaction that resulted in the nonlinearity of EPSP summation at distal dendrites is thus likely to be restricted to a narrower time window for the linearity increase than that at proximal dendrites. If synapse compartmentalization is the cause of a restricted time window, such a restriction should be altered when the compartmentalization of inputs is largely removed by considering the temporal summation of consecutive EPSPs at the same input. We indeed found an increased linearity of summation and an elevated spiking probability for consecutively summed EPSPs $(\Delta t=10 \mathrm{~ms})$ at a single distal input after LTP induction (Fig. $7 A-D$ ), in sharp contrast to that found for the summation of EPSPs from two independent inputs with the same $10 \mathrm{~ms}$ interval (Figs. 2, 5C). Additional studies with dendritic recording and biophysical modeling incorporating realistic ion channel conductances may help to elucidate the effect of arborization and the electrical compartmentalization of synapses on the property and plasticity of dendritic integration. 

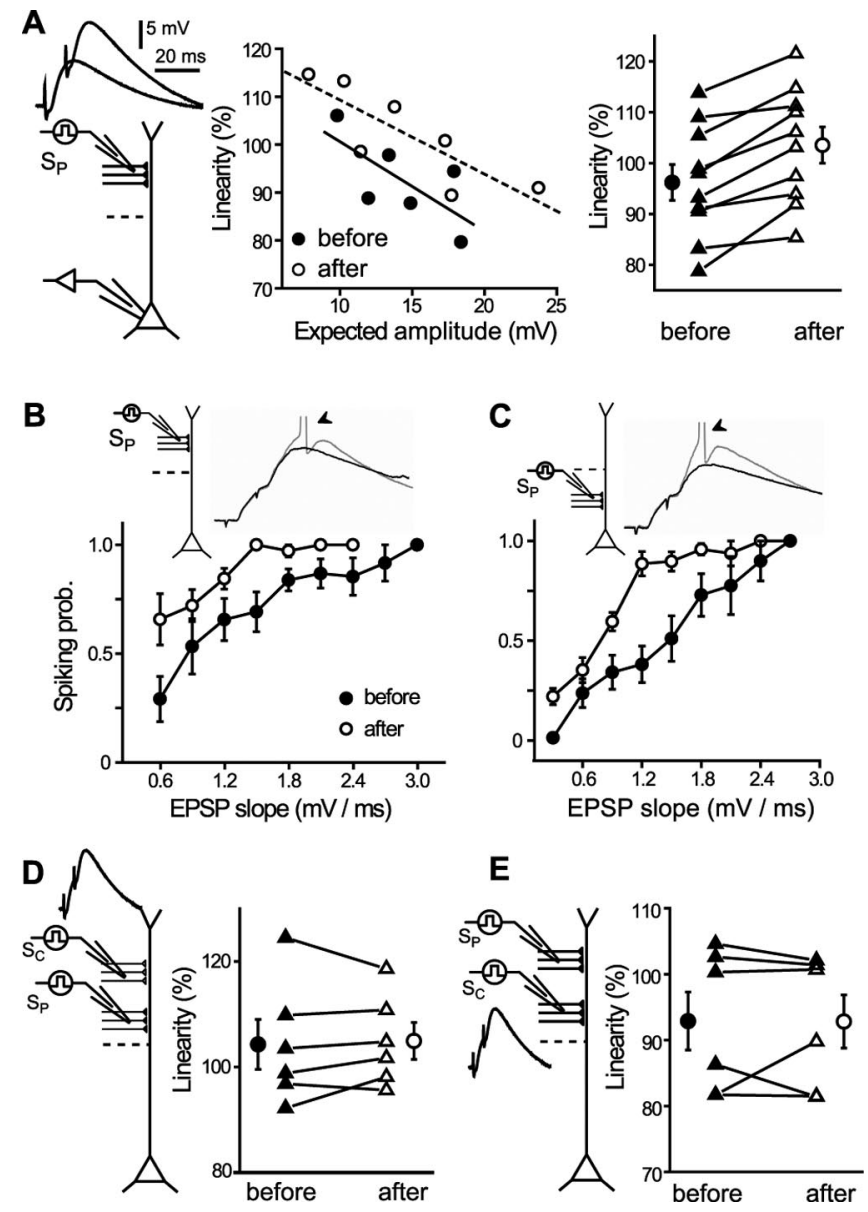

Figure 7. Increased linearity of temporal summation for asynchronous EPSPs elicited at a single distal input after LTP induction. $\boldsymbol{A}$, The linearity of summation of consecutive EPSPs (interval, $10 \mathrm{~ms}$ ) at a single distal input increased ( $p=0.0002 ; n=10$ ) after LTP induction at the same input. $\boldsymbol{B}, \boldsymbol{C}$, The spiking probability for summed EPSPs (interval, $10 \mathrm{~ms}$ ) at a single distal $(\boldsymbol{B})$ or proximal $(\boldsymbol{C})$ input was increased over a wide range of EPSP amplitudes after LTP induction. Traces depict sample of summed EPSPs before (dark) and after (gray) LTP induction. Arrowheads indicate truncated action potentials $[p=0.0067(\boldsymbol{B}) ; p=0.0026$ (C); MannWhitney $U$ test]. $\boldsymbol{D}, \boldsymbol{E}$, Input specificity in the enhancement of temporal summation after LTP induction. Regardless of the relative location of $S_{P}$ and $S_{C}, S_{P}$ proximal to $S_{C}(D)$, or $S_{P}$ distal to $S_{C}$ $(\boldsymbol{E})$, temporal summation at $\mathrm{S}_{\mathrm{C}}$ for two consecutive EPSPs (interval, $10 \mathrm{~ms}$ ) was not affected $[p=0.69(\boldsymbol{D}) ; p=0.97(\boldsymbol{E})]$.

\section{Functional implications}

Because of dendritic filtering, EPSPs elicited at distal inputs tend to be small in amplitude and prolonged in kinetics when reaching the soma, and their influence on the spiking output is likely to depend mainly on temporal integration (Segev, 1995; Magee, 2000). This may reduce the temporal precision in the information carried by the distal inputs. After LTP induction at the distal dendrite, we observed an enhancement of the spiking output by coincident distal inputs (Fig. 5). This suggests a mechanism for increasing the temporal fidelity of the information carried by the distal inputs. A previous report revealed that a mechanism for coincidence detection occurred at the distal dendrites of cortical pyramidal neurons through the generation of local dendritic spikes in the neocortical neuron (Williams and Stuart, 2002). The initiation of distal dendritic spikes has also been found only for highly coincident distal inputs in hippocampal CA1 neurons (Gasparini et al., 2004). Thus, it is likely that our observed linearity increase for summation of distal inputs arriving within a narrow time window after LTP induction (Fig. $4 A$ ) may promote the generation of distal dendritic spikes, which in turn will help coincidence detection. Moreover, feed-forward inhibition has also been found to participate in the coincident detection in CA1 pyramidal neurons by sharpening the EPSP waveform, which reduces the spiking probability of asynchronously summed EPSPs (Pouille and Scanziani, 2001); however, this may lead to a potential reduction in the temporal fidelity of SC-CA1 inputs when these inputs undergo LTP, unless the SC inputs to the feed-forward inhibitory interneurons were also potentiated simultaneously (Lamsa et al., 2005). In the present study, the selective enhancement of coincident detection of distal inputs associated with the potentiated inputs (Fig. 5) provides an additional mechanism to selectively increase the temporal fidelity of the information carried by the potentiated distal inputs. This may further indicate an experience-dependent mechanism for facilitating coincidence detection at the distal dendrites of pyramidal neurons (Williams and Stuart, 2002).

Because the increased postsynaptic spiking triggered by coincident inputs will facilitate further activity-dependent potentiation of these inputs by correlated presynaptic and postsynaptic spiking (Bi and Poo, 2001), the enhanced dendritic integration for coincident distal inputs described here suggests that distal inputs may be selectively costabilized by virtue of their synchronicity in exciting the same postsynaptic cell. This mechanism may work in concert with the distal-proximal difference in spike timing-dependent plasticity (Froemke et al., 2005), leading to dendritic location-specific input selection based on the temporal characteristics of presynaptic spike trains during the development of hippocampal connections.

At proximal inputs closer to the soma, EPSPs exhibit larger amplitude and faster kinetics; therefore, a few coincident inputs at the proximal dendrite are likely to evoke action potentials. The enhanced summation efficacy for both synchronous and asynchronous proximal inputs will further add to the weight of the potentiated input in controlling the neuronal firing without a compromise in its temporal fidelity. Together, our results showed that LTP induction is accompanied by a selective facilitation of coincidence detection for distal inputs, whereas temporal integration is selectively facilitated for proximal inputs.

In conclusion, our results demonstrated a marked activitydependent plasticity of dendritic functions, with the enhancement of dendritic integration exhibiting a striking dendritic location-dependent temporal selectivity, leading to differential promotion of coincidence detection and temporal integration at distal and proximal dendrites, respectively. Together with synaptic plasticity, this plasticity of dendritic functions constitutes an integral part of activity-dependent information processing and storage in neural circuits.

\section{References}

Agmon-Snir H, Carr CE, Rinzel J (1998) The role of dendrites in auditory coincidence detection. Nature 393:268-272.

Aizenman CD, Linden DJ (2000) Rapid, synaptically driven increases in the intrinsic excitability of cerebellar deep nuclear neurons. Nat Neurosci 3:109-111.

Bannister NJ, Larkman AU (1995) Dendritic morphology of CA1 pyramidal neurones from the rat hippocampus: I. Branching patterns. J Comp Neurol 360:150-160.

Bi G, Poo M (2001) Synaptic modification by correlated activity: Hebb's postulate revisited. Annu Rev Neurosci 24:139-166.

Bickmeyer U, Heine M, Manzke T, Richter DW (2002) Differential modulation of $\mathrm{I}(\mathrm{h})$ by 5 -HT receptors in mouse CA1 hippocampal neurons. Eur J Neurosci 16:209-218.

Bolshakov VY, Siegelbaum SA (1995) Regulation of hippocampal transmit- 
ter release during development and long-term potentiation. Science 269:1730-1734.

Brown TH, Kairiss EW, Keenan CL (1990) Hebbian synapses: biophysical mechanisms and algorithms. Annu Rev Neurosci 13:475-511.

Carr DB, Day M, Cantrell AR, Held J, Scheuer T, Catterall WA, Surmeier DJ (2003) Transmitter modulation of slow, activity-dependent alterations in sodium channel availability endows neurons with a novel form of cellular plasticity. Neuron 39:793-806.

Cash S, Yuste R (1999) Linear summation of excitatory inputs by CA1 pyramidal neurons. Neuron 22:383-394.

Colbert CM, Magee JC, Hoffman DA, Johnston D (1997) Slow recovery from inactivation of $\mathrm{Na}^{+}$channels underlies the activity-dependent attenuation of dendritic action potentials in hippocampal CA1 pyramidal neurons. J Neurosci 17:6512-6521.

Daoudal G, Hanada Y, Debanne D (2002) Bidirectional plasticity of excitatory postsynaptic potential (EPSP)-spike coupling in CA1 hippocampal pyramidal neurons. Proc Natl Acad Sci USA 99:14512-14517.

Engert F, Bonhoeffer T (1999) Dendritic spine changes associated with hippocampal long-term synaptic plasticity. Nature 399:66-70.

Frick A, Magee JC, Johnston D (2004) LTP is accompanied by an enhanced local excitability of pyramidal neuron dendrites. Nat Neurosci 7:126-135.

Froemke RC, Poo MM, Dan Y (2005) Spike-timing-dependent synaptic plasticity depends on dendritic location. Nature 434:221-225.

Gasparini S, Migliore M, Magee JC (2004) On the initiation and propagation of dendritic spikes in CAl pyramidal neurons. J Neurosci 24:11046-11056.

Hausser M, Spruston N, Stuart GJ (2000) Diversity and dynamics of dendritic signaling. Science 290:739-744.

Hoffman DA, Magee JC, Colbert CM, Johnston D (1997) $\mathrm{K}^{+}$channel regulation of signal propagation in dendrites of hippocampal pyramidal neurons. Nature 387:869-875.

Konig P, Engel AK, Singer W (1996) Integrator or coincidence detector? The role of the cortical neuron revisited. Trends Neurosci 19:130-137.

Lamsa K, Heeroma JH, Kullmann DM (2005) Hebbian LTP in feed-forward inhibitory interneurons and the temporal fidelity of input discrimination. Nat Neurosci 8:916-924.

Magee JC (1998) Dendritic hyperpolarization-activated currents modify the integrative properties of hippocampal CA1 pyramidal neurons. J Neurosci 18:7613-7624.

Magee JC (1999) Dendritic $I_{\mathrm{h}}$ normalizes temporal summation in hippocampal CA1 neurons. Nat Neurosci 2:508-514.

Magee JC (2000) Dendritic integration of excitatory synaptic input. Nat Rev Neurosci 1:181-190.

Magee JC, Cook EP (2000) Somatic EPSP amplitude is independent of synapse location in hippocampal pyramidal neurons. Nat Neurosci 3:895-903.

Magee JC, Johnston D (1997) A synaptically controlled, associative signal for Hebbian plasticity in hippocampal neurons. Science 275:209-213.
Malenka RC, Nicoll RA (1999) Long-term potentiation: a decade of progress? Science 285:1870-1874.

Maletic-Savatic M, Malinow R, Svoboda K (1999) Rapid dendritic morphogenesis in CA1 hippocampal dendrites induced by synaptic activity. Science 283:1923-1927.

Migliore M, Shepherd GM (2002) Emerging rules for the distributions of active dendritic conductances. Nat Rev Neurosci 3:362-370.

Nicoll RA, Malenka RC (1999) Expression mechanisms underlying NMDA receptor-dependent long-term potentiation. Ann NY Acad Sci 868:515-525.

Pouille F, Scanziani M (2001) Enforcement of temporal fidelity in pyramidal cells by somatic feed-forward inhibition. Science 293:1159-1163.

Rall W (1977) Cable theory for neurons. In: Handbook of physiology: the nervous system, Vol 1 (Eric RK, Brookhardt JM, Vernon BM, eds), pp 39-97. Baltimore: Williams \& Wilkins.

Rall W (1995) Theoretical significance of dendritic trees for neuronal inputoutput relations. In: The theoretical foundation of dendritic function (Segev I, Rinzel J, Shepard GM, eds), pp 122-146. Cambridge, MA: MIT.

Reyes A (2001) Influence of dendritic conductances on the input-output properties of neurons. Annu Rev Neurosci 24:653-675.

Segev I (1995) Introduction. In: The theoretical foundation of dendritic function (Segev I, Rinzel J, Shepard GM, eds), pp 117-121. Cambridge, MA: MIT.

Segev I, London M (2000) Untangling dendrites with quantitative models. Science 290:744-750.

Shadlen MN, Newsome WT (1994) Noise, neural codes and cortical organization. Curr Opin Neurobiol 4:569-579.

Toni N, Buchs PA, Nikonenko I, Bron CR, Muller D (1999) LTP promotes formation of multiple spine synapses between a single axon terminal and a dendrite. Nature 402:421-425.

Urban NN, Barrionuevo G (1998) Active summation of excitatory postsynaptic potentials in hippocampal CA3 pyramidal neurons. Proc Natl Acad Sci USA 95:11450-11455.

Wang Z, Xu NL, Wu CP, Duan S, Poo MM (2003) Bidirectional changes in spatial dendritic integration accompanying long-term synaptic modifications. Neuron 37:463-472.

Williams SR, Stuart GJ (2002) Dependence of EPSP efficacy on synapse location in neocortical pyramidal neurons. Science 295:1907-1910.

Yuste R, Bonhoeffer T (2001) Morphological changes in dendritic spines associated with long-term synaptic plasticity. Annu Rev Neurosci 24:1071-1089.

Zhang W, Linden DJ (2003) The other side of the engram: experiencedriven changes in neuronal intrinsic excitability. Nat Rev Neurosci 4:885-900.

Zhou Q, Homma KJ, Poo MM (2004) Shrinkage of dendritic spines associated with long-term depression of hippocampal synapses. Neuron 44: $749-757$. 